\title{
Thyroid-Stimulating Hormone, White Matter Hyperintensities, and Functional Outcome in Acute Ischemic Stroke Patients
}

\author{
Christopher O. Leonards ${ }^{a}$ Harald J. Schneider ${ }^{\mathrm{e}}$ Thomas G. Liman $^{\mathrm{a}, \mathrm{b}}$ \\ Jochen B. Fiebach ${ }^{a}$ Matthias Endres ${ }^{a-d}$ Martin Ebinger ${ }^{a, b}$ \\ ${ }^{a}$ Center for Stroke Research Berlin (CSB), b Klinik und Hochschulambulanz für Neurologie, \\ 'Excellence Cluster NeuroCure, and d German Center for Cardiovascular Research (DZHK), \\ Charité - Universitätsmedizin, Berlin, and ${ }^{~}$ Medizinische Klinik und Poliklinik IV, \\ Klinikum der Universität München, Munich, Germany
}

Key Words

Stroke $\cdot$ Ischemia $\cdot$ Hyperthyroidism · Hypothyroidism · Thyrotropin · White matter hyperintensities · Leukoaraiosis

\begin{abstract}
Background: Thyroid-stimulating hormone (TSH) concentrations are frequently altered in acute ischemic stroke patients. It is becoming increasingly apparent that various hormones in the hypothalamus-pituitary-thyroid axis may be associated with functional stroke outcome. We have previously shown that white matter hyperintensities (WMH) of presumed vascular origin are strong indicators of functional outcome. It is unclear whether an association exists between WMH and TSH. We therefore sought to determine whether TSH levels, measured in acute ischemic stroke patients, are associated with WMH and functional outcome. Methods: We analyzed all first ischemic stroke patients who participated in the Berlin 'Cream \& Sugar' Study (NCT 01378468) and completed a 1-year follow-up assessment from January 2009 to March 2013. Patients were stratified into 3 groups: (1) low TSH $(0.1-0.44 \mu \mathrm{U} / \mathrm{ml})$; (2) normal TSH $(0.44-2.5 \mu \mathrm{U} / \mathrm{ml})$, and (3) high TSH $(2.5-20 \mu \mathrm{U} / \mathrm{ml})$. WMH were assessed using the Fazekas and Wahlund visual rating scales. Functional outcome was assessed using the modified Rankin Scale and was performed via telephone at 1 year by a certified rater. Results: 183 patients were included [median age 66, interquartile range (IQR) 54-75; 33\% females; median National Institute of Health Stroke Scale 3, IQR 1-4, range 0-24]. Venous samples were collected a median of 4 days (IQR 3-5) following initial symptom onset between 8 and 9 a.m. following a 10-hour fast. Patients with normal TSH levels $(n=132 ; 72 \%)$ had significantly higher rates of prestroke diabetes than patients with high TSH levels (normal TSH 17\%; high TSH 1\%; $p=$ 0.03). Additionally, patients with normal TSH levels tended to have higher estimated glomerular filtration rates than patients with high and low TSH concentrations (normal TSH median
\end{abstract}

Christopher O. Leonards

Center for Stroke Research Berlin (CSB)

Charité - Universitätsmedizin Berlin, Campus Mitte, Charité Platz 1

DE-10117 Berlin (Germany)

E-Mail christopher.leonards@ charite.de 
Leonards et al.: Thyroid-Stimulating Hormone, White Matter Hyperintensities, and Functional Outcome in Acute Ischemic Stroke Patients

estimated glomerular filtration rates: $83 \mathrm{ml} / \mathrm{min} / 1.73 \mathrm{~m}^{2}$; high TSH median estimated glomerular filtration rates: $76 \mathrm{ml} / \mathrm{min} / 1.73 \mathrm{~m}^{2}$; low TSH median: $78 \mathrm{ml} / \mathrm{min} / 1.73 \mathrm{~m}^{2} ; \mathrm{p}=0.068$ ). Logistical regression analysis force-adjusted for age (quartiles), NIHSS (quartiles), prestroke diabetes status, and stroke subtype revealed significant associations between WMH and TSH [Wahlund scores: odds ratio 2.547, 95\% confidence interval (CI) 1.159-5.598, $\mathrm{p}=0.020$; Fazekas scores: odds ratio $2.530,95 \%$ CI 1.115-5.741, $p=0.003]$. Functional outcome was not significantly associated with TSH levels in univariate or multivariate models. Conclusion: TSH levels are independently associated with $\mathrm{WMH}$ in acute ischemic stroke patients. Based on our findings, we cannot recommend assessing TSH to estimate the 1-year functional outcome following ischemic stroke.

(C) 2014 S. Karger AG, Basel

\section{Introduction}

Thyroid-stimulating hormone (TSH) concentrations are frequently altered in critically ill patients, specifically during the acute period of their illness [1]. During the early recovery period following ischemic stroke, thyroid dysfunction has been noted in more than $36 \%$ of patients [2]. It is becoming increasingly apparent that various hormones in the hypothalamuspituitary-thyroid axis, specifically triiodothyronine, may be associated with functional stroke outcome [3-6]. Data regarding TSH levels are less clear. Some studies have found that thyroid dysfunction (either clinical or subclinical) is associated with functional outcome following ischemic stroke $[3,5,7]$, whereas others have not found this $[8,9]$.

White matter hyperintensities (WMH) of presumed vascular origin [10] have been shown to be strong indicators of functional outcome [11,12] and stroke recurrence [13]. It is unclear, however, whether an association exists between WMH and TSH in acute ischemic stroke patients. Because central nervous system tissue, oligodendrocytes in particular, is known to rely heavily on thyroid hormones for embryonic differentiation, maintenance, and myelinization [14], it is possible that altered TSH levels in the acute period of ischemic stroke represent a possible modulator of WMH and functional outcome.

We therefore sought to determine whether TSH levels are associated with WMH and functional outcome in acute ischemic stroke patients.

\section{Methods and Methods}

\section{Participants}

This was a retrospective subanalysis of the Berlin 'Cream \& Sugar' Study (NCT 01378468). The Berlin 'Cream \& Sugar' Study is an ongoing prospective cohort study seeking to determine whether elevated postchallenge triglycerides are associated with recurrent stroke. Detailed methods are presented elsewhere [15]. Briefly, we screened all male and female first-time acute ischemic stroke patients over 18 years of age (3-7 days after symptom onset), who were admitted to one of three university campus hospitals in Berlin. Because thyroid dysfunction was not an exclusion criterion for participating in the 'Cream \& Sugar' Study, we excluded all 'Cream \& Sugar' participants who were taking L-thyroxine for the purpose of this analysis. Ischemic stroke was defined as a focal neurological deficit lasting for at least $24 \mathrm{~h}$ with no signs of hemorrhage on cerebral imaging. All suspected ischemic strokes were verified radiologically using diffusion-weighted magnetic resonance imaging. Strokes were categorized according to a mechanism-based classification scheme (Trial of ORG 10172 in Acute Stroke Treatment or TOAST) [16]. Stroke severity was assessed on hospital admission and at the 

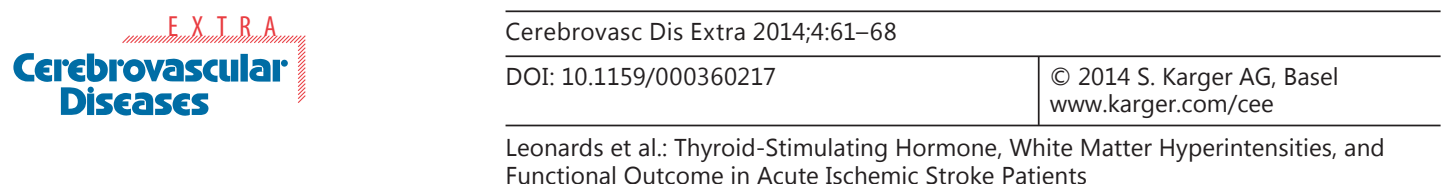

time of testing using the National Institute of Health Stroke Scale (NIHSS), the modified Rankin Scale (mRS), and the Barthel Index. Functional outcome was assessed via telephone follow-up after 1 year using the mRS and the Barthel Index and was performed by a certified rater. All patients provided informed consent, and the study was approved by the local ethics committee.

\section{Blood Samples}

Baseline data and venous blood samples were collected after participants had provided informed consent and fasted overnight for $\geq 12 \mathrm{~h}$. Fasting blood samples were drawn at 8 a.m. Triglyceride and cholesterol concentrations were enzymatically determined in freshly drawn venous blood samples using a Cobas 6000 analyzer (Roche/Hitachi). The glomerular filtration rate was estimated using the Modification of Diet in Renal Disease [17] formula according to the relationship: $186 \times$ serum creatinine ${ }^{-1.154} \times$ age $^{-0.203} \times 1.210$ (if black) $\times 0.742$ (if female) . Diabetes was defined as the current use of antidiabetic medication, a serum glycosylated hemoglobin of $>6.5 \%$ or a 2 -hour oral glucose tolerance test value of $>200 \mathrm{mg} / \mathrm{dl}$. Hypertension was defined as current antihypertensive medication use. Hyperlipidemia was defined as fasting total cholesterol $\geq 200 \mathrm{mg} / \mathrm{dl}$ and/or fasting triglycerides $\geq 150 \mathrm{mg} / \mathrm{dl}$ and/or the use of lipid-lowering medications prior to ischemic stroke [18].

\section{Combined Oral Triglyceride and Glucose Tolerance Test}

Fasting blood samples were drawn at 8 a.m. Upon completion of phlebotomy, patients drank $250 \mathrm{ml}$ of $32 \%$ fat cream within $30 \mathrm{~min}$ in the presence of a Center for Stroke Research Berlin staff member to ensure that the cream was ingested. Three hours later (11 a.m.), a second blood draw was performed immediately followed by a standard 75-gram oral glucose tolerance test. Subsequent blood draws were then performed at 12 and 1 p.m.

\section{Image Acquisition and WMH}

MRI was performed using both 3-tesla (Tim Trio; Siemens, Erlangen, Germany) and 1.5-tesla (Avanto, Siemens Medical Solutions, Erlangen, Germany) scanners. FLAIR or T2-weighted images were used to assess WMH. WMH were scored according to both the agerelated white matter changes [19] and Fazekas visual classification scales [20]. In the agerelated white matter changes system, FLAIR and T2 image hyperintensities are rated from 0 to 3 based on size and confluence of the lesions on both the right and left sides of the brain in the following prespecified regions: frontal, parieto-occipital, temporal, infratentorial/cerebellum, and basal ganglia. The final score is the sum of all regions and ranges from 0 (no WMH) to 30 (most severe WMH). In the Fazekas classification system, the image with the most severe $\mathrm{WMH}$ is rated on a scale of $0-3(0=$ no $\mathrm{WMH} ; 1=$ punctate foci; $2=$ beginnings of confluent foci, and $3=$ large confluent areas).

\section{Statistical Analysis}

We stratified patients into 3 groups according to TSH levels [(1) low TSH $(0.1-\mu \mathrm{U} / \mathrm{ml}) ;(2)$ normal TSH $(0.44-2.5 \mu \mathrm{U} / \mathrm{ml})$, and (3) high TSH $(2.5-20 \mu \mathrm{U} / \mathrm{ml})]$ and according to follow-up mRS scores (mRS: 0-1,2-3, and 4-6). Continuous variables were tested for normality of distribution using the Kolmogorov-Smirnov test. Kruskal-Wallis tests were used to examine relationships between baseline and serum parameters (not normally distributed) and TSH groups. The relationship between categorical variables was assessed using two-sided Fisher exact tests. If a significant relationship was found, post hoc analyses using Sidak's method were performed (level of significance at 0.017). For multivariate models, variables that were not normally distributed (i.e. age and NIHSS) were stratified into quartiles. Wahlund scores were stratified into tertiles (Wahlund score $0-4=\mathrm{low} /$ no WMH; Wahlund score $5-10=$ moderate $\mathrm{WMH}$, and Wahlund $>10=$ severe $\mathrm{WMH}$ ). Ordinal logistical regression analysis models were 
Leonards et al.: Thyroid-Stimulating Hormone, White Matter Hyperintensities, and Functional Outcome in Acute Ischemic Stroke Patients

force-adjusted for age, NIHSS scores, prestroke diabetes status, and stroke subtype (TOAST criteria). To assess the potential relationship between WMH and TSH, we generated 4 models: namely, model 1 included stratified Wahlund scores as the independent variable, model 2 included Fazekas scores as the independent variable, model 3 included deep WMH as the independent variable, and model 4 included periventricular WHM as the independent variable. Stratified TSH concentrations were used as the dependent variable in models 1-4. To determine whether an association existed between the follow-up functional outcome and TSH concentrations, we performed an ordinal logistical regression analysis force-adjusted for age, NIHSS, and stroke subtype, using follow-up mRS scores as the independent variable and stratified TSH concentrations as the dependent variable. Thereafter, a retrospective analysis was conducted that included patients taking L-thyroxine. A further retrospective binary logistical regression analysis was performed to determine whether recurrent cerebrovascular events (stroke or TIA), new cardiovascular events/symptoms (myocardial infarction or angina pectoris), or the development of comorbidities between the time of initial testing and the 1-year follow-up influenced our results concerning functional outcome. This analysis was adjusted for age (quartiles), new cardiovascular, or cerebrovascular events (combined variable including myocardial infarction, angina pectoris, recurrent stroke, and TIA between the time of testing and follow-up), death, follow-up hospitalization (for stroke or other causes), new neurological deficit (where patients sought treatment), and included TSH levels (normal range $=$ reference) and Wahlund scores. Unless otherwise specified, the significance was set at $\mathrm{p} \leq 0.05$. All statistical analyses were 2-tailed and conducted using SPSS 19.

\section{Results}

The Berlin 'Cream \& Sugar' Study tested 248 participants between January 2009 and March 2013. Of these, 197 patients completed the follow-up, and of these, 183 had complete datasets and were eligible for inclusion in this retrospective analysis. Baseline characteristics of the patient sample are presented in table 1. Six deaths (1 patient with low TSH levels, 2 patients with high TSH levels, and 3 patients with normal TSH levels) occurred before the 1-year follow-up. Patients with normal TSH levels had significantly higher rates of diabetes than patients with high TSH levels (Sidak's post hoc method, $p=0.03$ ). Diastolic blood pressure significantly increased across the groups (table 1).

In the univariate analysis that excluded all patients taking L-thyroxine, low TSH levels (normal TSH levels = reference) were not significantly associated with outcome regardless of mRS stratification [mRS excellent: unadjusted odds ratio $2.0,95 \%$ confidence interval (CI) 0.423-9.199, $\mathrm{p}=0.446$; mRS good: unadjusted odds ratio $1.6,95 \%$ CI $0.290-8.649, \mathrm{p}=0.633$; mRS not stratified: unadjusted odds ratio $1.3,95 \%$ CI $0.654-2.529, \mathrm{p}=0.466]$. Functional outcomes did not significantly differ between patients with normal (reference category) and high TSH levels (mRS excellent: unadjusted odds ratio 0.888, 95\% CI 0.426-1.850, p = 0.853; mRS good: unadjusted odds ratio $0.990,95 \%$ CI $0.406-2.12, \mathrm{p}=1.00$; mRS not stratified: unadjusted odds ratio $0.944,95 \%$ CI $0.499-1.789, \mathrm{p}=0.861$ ). Including patients taking L-thyroxine did not change the results.

The ordinal logistical regression analysis force-adjusted for age, NIHSS scores, prestroke diabetes status, and stroke subtype (TOAST criteria) revealed that TSH levels independently associated with WMH severity (Wahlund scores: odds ratio 2.547, 95\% CI 1.159-5.598, p = 0.020; Fazekas scores: odds ratio $2.530,95 \%$ CI 1.115-5.741, $\mathrm{p}=0.003$ ). The ordinal logistical regression analysis force-adjusted for age, NIHSS scores, and stroke subtype (TOAST criteria) revealed that there was no significant association between the 1-year functional outcome and TSH (adjusted odds ratio 1.292, 95\% CI 0.811-2.058, $\mathrm{p}=0.281$ ). 


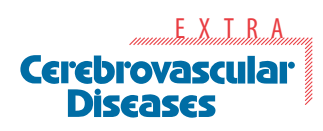

\begin{tabular}{l|l}
\hline \multicolumn{2}{l}{ Cerebrovasc Dis Extra 2014;4:61-68 } \\
\hline DOI: 10.1159/000360217 & $\begin{array}{l}\text { (C) 2014 S. Karger AG, Basel } \\
\text { www.karger.com/cee }\end{array}$ \\
\hline
\end{tabular}

Leonards et al.: Thyroid-Stimulating Hormone, White Matter Hyperintensities, and Functional Outcome in Acute Ischemic Stroke Patients

Table 1. Demographic characteristics according to the TSH level

\begin{tabular}{|c|c|c|c|c|}
\hline & High TSH & Normal TSH & Low TSH & $\mathrm{p}$ value \\
\hline Total participants, $\mathrm{n}$ & 41 & 132 & 10 & \\
\hline Females, $\mathrm{n}(\%)^{\mathrm{a}}$ & $13(7)$ & $45(25)$ & $6(3)$ & 0.239 \\
\hline Age, years ${ }^{b}$ & $62(51-75)$ & $67(57-75)$ & $68(57-80)$ & 0.613 \\
\hline L-Thyroxine, n (\%) ${ }^{\mathrm{a}}$ & $1(0.5)^{c}$ & $8(4.4)^{c}$ & $3(1.6)^{c}$ & $0.021^{\mathrm{c}}$ \\
\hline Atrial fibrillation, $\mathrm{n}(\%)^{\mathrm{a}}$ & $3(2)$ & $17(12)$ & $2(1)$ & 0.675 \\
\hline Hypertension, $\mathrm{n}(\%)^{\mathrm{a}}$ & $22(13)$ & $81(48)$ & $9(67)$ & 0.115 \\
\hline Hyperlipidemia, n (\%) & $13(8)$ & $37(23)$ & $2(1)$ & 0.740 \\
\hline Current smokers, $\mathrm{n}(\%)^{\mathrm{a}}$ & $9(5)$ & 31 (19) & $1(0.6)$ & 0.641 \\
\hline Diabetes, n (\%) & $2(1)$ & $28(17)$ & $1(0.6)$ & 0.019 \\
\hline Days since stroke & $5(3-6)$ & $4(3-5)$ & $4(3-5)$ & 0.167 \\
\hline NIHSS (admission) ${ }^{\mathrm{b}}$ & $3(2-7)$ & $3(2-4)$ & $2(0-4)$ & 0.402 \\
\hline mRS (time of testing) & $1(0-2)$ & $1(1-2)$ & $1(0-2)$ & 0.594 \\
\hline BI (time of testing) & $100(90-100)$ & $100(90-100)$ & $100(90-100)$ & 0.903 \\
\hline eGFR ${ }^{b}$ & $76(63-95)$ & $83(73-97)$ & $78(44-89)$ & 0.068 \\
\hline Wahlund score ${ }^{\mathrm{b}}$ & $2(0-3)$ & $3(1-8)$ & $3(1-4)$ & 0.037 \\
\hline Severe WMH, n (\%) ${ }^{\mathrm{a}}$ & $3(2)$ & $14(9)$ & $0(0)$ & 0.243 \\
\hline Severe DWMH, n (\%) & $1(0.7)$ & $10(7)$ & $0(0)$ & 0.570 \\
\hline Severe PVWMH, n (\%) ${ }^{\mathrm{a}}$ & $3(2)$ & $13(9)$ & $0(0)$ & 0.836 \\
\hline Microbleeds, n (\%) $)^{\mathrm{a}}$ & $0(0)$ & $6(4)$ & $2(1)$ & 0.061 \\
\hline Creatinine, $\mathrm{mg} / \mathrm{dl}^{\mathrm{b}}$ & $0.98(0.87-1.14)$ & $0.89(0.76-1.03)$ & $0.94(0.72-1.29)$ & 0.076 \\
\hline Systolic BP & $130(120-150)$ & $137(120-150)$ & $149(120-157)$ & 0.427 \\
\hline Diastolic $\mathrm{BP}^{\mathrm{b}}$ & $70(69-80)$ & $80(70-85)$ & $80(75-91)$ & 0.020 \\
\hline $\mathrm{BMI}^{\mathrm{b}}$ & $27(24-28)$ & $26(24-29)$ & $29(27-34)$ & 0.145 \\
\hline Total cholesterol, mg/dl ${ }^{b}$ & $180(153-215)$ & $182(157-210)$ & $196(135-238)$ & 0.787 \\
\hline $\mathrm{LDL}, \mathrm{mg} / \mathrm{dl}^{\mathrm{b}}$ & $106(88-140)$ & $109(88-137)$ & $123(70-155)$ & 0.950 \\
\hline $\mathrm{CRP}, \mathrm{mg} / \mathrm{dl}^{\mathrm{b}}$ & $0.23(0.1-0.69)$ & $0.27(0.12-0.65)$ & $0.37(0.09-0.72)$ & 0.783 \\
\hline $\mathrm{HbA}_{1 \mathrm{c}}, \% \mathrm{~b}$ & $5.6(5.2-6.0)$ & $5.7(5.3-6.2)$ & $5.8(5.3-6.5)$ & 0.740 \\
\hline Waist-to-hip ratio ${ }^{\mathrm{b}}$ & $0.97(0.94-1.0)$ & $0.97(0.92-1.0)$ & $0.96(0.89-1.0)$ & 0.777 \\
\hline Glucose, $\mathrm{mg} / \mathrm{dl}^{\mathrm{b}}$ & $94(86-103)$ & $97(87-112)$ & $99(90-132)$ & 0.135 \\
\hline Triglycerides, mg/dl ${ }^{b}$ & $115(86-171)$ & $118(90-151)$ & $97(71-135)$ & 0.394 \\
\hline Follow-up mRS & $1(0-2)$ & $1(0-2)$ & $2(0-3)$ & 0.816 \\
\hline mRS deterioration, $\mathrm{n}(\%)^{\mathrm{a}}$ & $14(11)$ & $33(26)$ & $4(3)$ & 0.649 \\
\hline Follow-up BI & $100(98-100)$ & $100(95-100)$ & $100(100-100)$ & 0.774 \\
\hline Stroke subtype, n (\%) & & & & 0.112 \\
\hline Large artery & $12(7)$ & $37(21)$ & $3(2)$ & \\
\hline Cardioembolic & $7(4)$ & $25(14)$ & $2(1)$ & \\
\hline Small artery & $5(3)$ & $39(22)$ & $1(1)$ & \\
\hline Other determined subtype & $6(3)$ & $8(5)$ & $2(1)$ & \\
\hline Undetermined subtype & $11(6)$ & $20(11)$ & $1(1)$ & \\
\hline
\end{tabular}

Values in parentheses represent median (IQR) unless otherwise specified. BI = Barthel Index; $\mathrm{BP}=$ blood pressure; CRP = C-reactive protein; DWMH = deep WMH; eGFR = estimated glomerular filtration rate; $\mathrm{HbA}_{1 \mathrm{c}}=$ glycosylated hemoglobin; $\mathrm{LDL}=$ low-density lipoprotein; $\mathrm{mRS}$ deterioration $=\mathrm{mRS}$ score at the time of testing versus mRS score at the follow-up interview (classified as same, improvement, or deterioration); NIHSS = National Institute of Health Stroke Scale; PVWMH = periventricular WMH. ${ }^{a}$ Fisher's exact test followed by Sidak's post hoc method (if significant). ${ }^{\mathrm{b}}$ Mann-Whitney U test. ${ }^{\mathrm{c}}$ All patients taking L-thyroxine were excluded from the initial analyses. Only the retrospective analysis included these patients.

The retrospective univariate analysis (Sidak's post hoc method) taking postchallenge glucose and triglyceride values into account revealed that the patients with high TSH levels had significantly lower 1-hour postchallenge glucose levels than those patients with normal TSH levels [high TSH: median glucose $125 \mathrm{mg} / \mathrm{dl}$, interquartile range (IQR) 92-170; normal TSH: median glucose $152 \mathrm{mg} / \mathrm{dl}$, IQR 114-184; $\mathrm{p}=0.016]$. No difference was observed across 
Leonards et al.: Thyroid-Stimulating Hormone, White Matter Hyperintensities, and Functional Outcome in Acute Ischemic Stroke Patients

TSH groups and 2-hour postchallenge glucose values. Levels of mean plasma glucose (MPG) tended to be lower in patients with high TSH levels than in those patients with normal TSH levels (high TSH: median MPG 107 mg/dl, IQR 90-129; normal TSH: median MPG 119 mg/dl, IQR 102-140; $p=0.072$ ). Additionally, MPG levels tended to be lower in patients with high TSH levels than in those with low TSH levels (high TSH: median MPG 107 mg/dl, IQR 90-129; low TSH: median MPG $135 \mathrm{mg} / \mathrm{dl}$, IQR 119-138; $\mathrm{p}=0.091$ ). Of the 3 postchallenge time points where triglycerides were measured, only 5 -hour postchallenge triglyceride levels significantly differed across the TSH groups. Patients with high TSH levels had significantly higher postchallenge triglycerides than patients with low TSH levels (high TSH: median 5-hour triglycerides 233 mg/dl, IQR 178-331; low TSH: median 5-hour triglycerides 133 mg/dl, IQR 120-186; $\mathrm{p}=0.036$ ). Finally, patients with low TSH levels tended to have lower postchallenge triglycerides than patients with normal TSH levels (low TSH: median 5-hour triglycerides $133 \mathrm{mg} /$ dl, IQR 120-186; normal TSH: median 5-hour triglycerides $213 \mathrm{mg} / \mathrm{dl}$, IQR 160-300; p = 0.075).

The retrospective forward-stepping binary logistical regression analysis adjusted for age (quartiles), new cardiovascular or cerebrovascular events [combined variable including myocardial infarction $(n=2)$, angina pectoris $(n=20)$, recurrent stroke and TIA $(n=13)$ between the time of testing and follow-up], death $(n=6)$, follow-up hospitalization [for stroke $(n=10)$ or other causes $(n=43)]$, and new neurological deficits [where patients sought treatment $(\mathrm{n}=34)]$ revealed that severe WMH was independently associated with 1-year functional disability ( $\mathrm{mRS} \geq 2$; Wahlund scores $\geq 10$ : odds ratio 9.375, 95\% CI 1.957-44.910, $\mathrm{p}=0.005$; Wahlund scores 5-9: odds ratio 1.607, 95\% CI 0.355-7.276, $\mathrm{p}=0.538$ ). Cardiovascular and cerebrovascular events (modeled as a combined group to decrease covariates) were also significantly and independently associated with the 1-year functional outcome (odds ratio 6.137, 95\% CI 1.388-27.139, $\mathrm{p}=0.017$ ). The TSH levels were not associated with the 1-year functional outcome.

\section{Discussion}

The primary findings of this study were (1) that TSH levels assessed in acute ischemic stroke patients are independently associated with WMH severity and (2) that they were not associated with the 1-year functional outcome. In accordance with previous findings [2], roughly $28 \%$ of our ischemic stroke patients had TSH concentrations outside the reference range. Interestingly, patients with normal TSH values had both higher rates of prestroke diabetes and higher postchallenge glucose levels than those patients with high TSH levels. Conversely, patients with high TSH levels had higher postchallenge triglyceride levels than patients with low TSH levels.

These findings support a growing body of literature showing that thyroid hormones play a role in both lipid regulation and the metabolic syndrome [21-23]. The possible role that TSH plays in nonfasting glucose and triglyceride regulation may help to explain the relationship we observed between WMH and TSH levels. Although the underlying pathophysiological mechanism of WMH remains unclear, it seems likely that diabetes and glucose play a contributing role [24]. We have also previously reported that there was a significant positive relationship between glycosylated hemoglobin levels and WMH [11].

Our results concerning TSH levels and functional outcome must be interpreted very cautiously, as it is likely that we were underpowered to detect such relationships. Based on the effect sizes that we found here (low TSH vs. normal TSH levels, Cohen's $d=0.236$, effect size $r=-0.1176$ ), we would have needed a total sample size of approximately 780 patients to achieve a power of $80 \%$. A recent study [5] reported that subclinical hyperthyroidism was 
Leonards et al.: Thyroid-Stimulating Hormone, White Matter Hyperintensities, and Functional Outcome in Acute Ischemic Stroke Patients

significantly associated with the 3-month functional outcome. However, the sample size in this study was similar to ours. Therefore, the conflicting results may be explained by more than simply power. First, the time to follow-up differed between the two studies (3 months vs. 1 year). Because subclinical hyperthyroid patients frequently (upwards of 50\%) and spontaneously revert to the euthyroid state over time (1-4 years), it is possible that the conflicting results may be explained by compensatory changes that occurred after 3 months $[25,26]$. Second, because we analyzed TSH levels in our acute ischemic stroke patients and had no data on free thyroid hormones ( $\mathrm{fT}_{3}$ and $\mathrm{fT}_{4}$ ), we could not differentiate between patients with overt and subclinical thyroid dysfunction, and this is a limitation of our study.

Our study has further limitations. As a Berlin 'Cream \& Sugar' substudy, inclusion criteria mandates [for inclusion criteria, see 15] resulted in a patient sample with relatively mild ischemic strokes (median NIHSS 3, IQR 0-4, range 0-24). Additionally, women were underrepresented in our patient sample (33\%). Because TSH levels may vary in the acute period of critical illness [1], it is possible that data leveraged from our mild acute ischemic stroke patient sample are not representative, specifically in patients that have had more severe strokes. We had no data on thyroid hormones $\left(\mathrm{fT}_{3}\right.$ and $\left.\mathrm{fT}_{4}\right)$ and therefore could not conclude whether high/low TSH levels are associated with thyroid dysfunction. Because we did not have information regarding TSH levels prior to stroke onset, it remains unclear whether the high/low TSH levels observed in our study were stroke-induced or feedback changes due to primary thyroid dysfunction. Further studies are needed to help determine whether TSH levels impact stroke outcome and WMH development/progression or vice versa. Finally, because we chose to follow patients for 1 year, it is possible that the development of comorbidities, such as recurrent stroke, has influenced our results concerning functional outcome. However, we observed no change from our initial findings following a retrospective analysis adjusted for these factors.

In conclusion, we found an independent association between WMH and TSH in acute ischemic stroke patients. Although WMH have been shown to be strongly associated with the 1-year functional outcome, we did not observe an association between TSH levels assessed in the acute ischemic stroke period and the 1-year functional outcome. We therefore cannot recommend assessing TSH to estimate the 1-year functional outcome following ischemic stroke.

\section{Acknowledgements}

The research leading to these results received funding from the Federal Ministry of Education and Research via the grant Center for Stroke Research Berlin (01 E0 0801), from the Volkswagen Foundation (Lichtenberg program to M. Endres), the German Center for Cardiovascular Research (DZHK) Charité (to M. Endres), the DFG (NeuroCure), and the EU (European Stroke Network).

\section{Disclosure Statement}

C.O.L., M. Ebinger, H.J.S., and T.G.L. have no conflicts of interest to declare. J.B.F. has received consultant or lecture fees from Boehringer Ingelheim, Lundbeck, Siemens, Sygnis, and Synarc. M. Endres has received grant support from AstraZeneca and Sanofi, has participated in advisory board meetings of Bayer, Boehringer Ingelheim, Bristol-Myers Squibb, MSD, Pfizer, Sanofi and has received honoraria from AstraZeneca, Bayer, Berlin Chemie, Bristol-Myers Squibb, Boehringer Ingelheim, Desitin, Eisei, Ever, GlaxoSmithKline, MSD, Novartis, Pfizer, Sanofi, Takeda, and Trommsdorff. 


\section{Cerebrovasculal Diseases}

\begin{tabular}{l|l}
\hline \multicolumn{2}{l}{ Cerebrovasc Dis Extra 2014;4:61-68 } \\
\hline DOI: 10.1159/000360217 & $\begin{array}{l}\text { ( 2) 2014 S. Karger AG, Basel } \\
\text { www.karger.com/cee }\end{array}$ \\
\hline
\end{tabular}

Leonards et al.: Thyroid-Stimulating Hormone, White Matter Hyperintensities, and Functional Outcome in Acute Ischemic Stroke Patients

\section{References}

1 Warner MH, Beckett GJ: Mechanisms behind the non-thyroidal illness syndrome: an update. J Endocrinol 2010;205:1-13.

2 Dimopoulou I, Kouyialis AT, Orfanos S, Armaganidis A, Tzanela M, Thalassinos N, et al: Endocrine alterations in critically ill patients with stroke during the early recovery period. Neurocrit Care 2005;3:224-229.

-3 Akhoundi FH, Ghorbani A, Soltani A, Meysamie A: Favorable functional outcomes in acute ischemic stroke patients with subclinical hypothyroidism. Neurology 2011;77:349-354.

4 Bunevicius A, Kazlauskas H, Raskauskiene N, Janusonis V, Bunevicius R: Ischemic stroke functional outcomes are independently associated with C-reactive protein concentrations and cognitive outcomes with triiodothyronine concentrations: a pilot study. Endocrine 2014;45:213-220.

-5 Wollenweber FA, Zietemann V, Gschwendtner A, Opherk C, Dichgans M: Subclinical hyperthyroidism is a risk factor for poor functional outcome after ischemic stroke. Stroke 2013;44:1446-1448.

6 Alevizaki M, Synetou M, Xynos K, Pappa T, Vemmos KN: Low triiodothyronine: a strong predictor of outcome in acute stroke patients. Eur J Clin Invest 2007;37:651-657.

7 Alevizaki M, Synetou M, Xynos K, Alevizaki CC, Vemmos KN: Hypothyroidism as a protective factor in acute stroke patients. Clin Endocrinol (Oxf) 2006;65:369-372.

-8 Rodondi N, Newman AB, Vittinghoff E, de Rekeneire N, Satterfield S, Harris TB, et al: Subclinical hypothyroidism and the risk of heart failure, other cardiovascular events, and death. Arch Intern Med 2005;165: 2460-2466.

-9 Imaizumi M, Akahoshi M, Ichimaru S, Nakashima E, Hida A, Soda M, et al: Risk for ischemic heart disease and all-cause mortality in subclinical hypothyroidism. J Clin Endocrinol Metab 2004;89:3365-3370.

10 Wardlaw JM, Smith EE, Biessels GJ, Cordonnier C, Fazekas F, Frayne R, et al: Neuroimaging standards for research into small vessel disease and its contribution to ageing and neurodegeneration. Lancet Neurol 2013; 12:822-838.

11 Leonards CO, Ipsen N, Malzahn U, Fiebach JB, Endres M, Ebinger M: White matter lesion severity in mild acute ischemic stroke patients and functional outcome after 1 year. Stroke 2012;43:3046-3051.

12 Henninger N, Lin E, Baker SP, Wakhloo AK, Takhtani D, Moonis M: Leukoaraiosis predicts poor 90-day outcome after acute large cerebral artery occlusion. Cerebrovasc Dis 2012;33:525-531.

13 Melkas S, Sibolt G, Oksala NKJ, Putaala J, Pohjasvaara T, Kaste M, et al: Extensive white matter changes predict stroke recurrence up to 5 years after a first-ever ischemic stroke. Cerebrovasc Dis 2012;34:191-198.

14 Schweizer U, Köhrle J: Function of thyroid hormone transporters in the central nervous system. Biochim Biophys Acta 2013;1830:3965-3973.

15 Ebinger M, Heuschmann PU, Jungehuelsing GJ, Werner C, Laufs U, Endres M: The Berlin 'Cream \& Sugar' Study: the prognostic impact of an oral triglyceride tolerance test in patients after acute ischaemic stroke. Int J Stroke 2010;5:126-130.

-16 Adams HP, Bendixen BH, Kappelle LJ, Biller J, Love BB, Gordon DL, et al: Classification of subtype of acute ischemic stroke. Definitions for use in a multicenter clinical trial. TOAST. Trial of Org 10172 in Acute Stroke Treatment. Stroke 1993;24:35-41.

17 National Kidney Foundation: K/DOQI clinical practice guidelines for chronic kidney disease: evaluation, classification, and stratification. Am J Kidney Dis 2002;39(2 suppl 1):S1-S266.

18 Lee T-H, Hsu W-C, Chen C-J, Chen S-T: Etiologic study of young ischemic stroke in Taiwan. Stroke 2002;33: 1950-1955.

19 Wahlund LO, Barkhof F, Fazekas F, Bronge L, Augustin M, Sjögren M, et al: A new rating scale for age-related white matter changes applicable to MRI and CT. Stroke 2001;32:1318-1322.

20 Fazekas F, Kleinert R, Offenbacher H, Schmidt R, Kleinert G, Payer F, et al: Pathologic correlates of incidental MRI white matter signal hyperintensities. Neurology 1993;43:1683-1689.

21 Asvold BO, Vatten LJ, Nilsen TIL, Bjøro T: The association between TSH within the reference range and serum lipid concentrations in a population-based study. The HUNT Study. Eur J Endocrinol 2007;156:181-186.

-22 Michalopoulou G, Alevizaki M, Piperingos G, Mitsibounas D, Mantzos E, Adamopoulos P, et al: High serum cholesterol levels in persons with 'high-normal' TSH levels: should one extend the definition of subclinical hypothyroidism? Eur J Endocrinol 1998;138:141-145.

23 Ruhla S, Weickert MO, Arafat AM, Osterhoff M, Isken F, Spranger J, et al: A high normal TSH is associated with the metabolic syndrome. Clin Endocrinol (Oxf) 2010;72:696-701.

-24 Gouw A, van der Flier WM, Fazekas F, van Straaten ECW, Pantoni L, Poggesi A, et al: Progression of white matter hyperintensities and incidence of new lacunes over a 3-year period: the Leukoaraiosis and Disability study. Stroke 2008;39:1414-1420.

25 Schultz M, Kistorp C, Raymond I, Dimsits J, Tuxen C, Hildebrandt P, et al: Cardiovascular events in thyroid disease: a population based, prospective study. Horm Metab Res 2011;43:653-659.

26 Parle JV, Franklyn JA, Cross KW, Jones SC, Sheppard MC: Prevalence and follow-up of abnormal thyrotrophin (TSH) concentrations in the elderly in the United Kingdom. Clin Endocrinol (Oxf) 1991;34:77-83. 